many physical properties and to modern techniques such as ESCA, EXAFS and DTA. There are some errors in the coverage of topics which are more often the province of physics. Most seriously, the process described for electrical conduction in metals appears to require thermal activation; another example is that superconductors are given the familiar value of -1 for their magnetic susceptibility, but this is not correct for the old-fashioned system in which susceptibility is defined in the book.

L. Solymar and D. Walsh's book is for students of engineering who need to know about the materials used in devices. The fourth edition of their Lectures on the Electrical Properties of Materials includes two new chapters which extend the subject range to include optical as well as electrical devices. The style of writing has the informality hinted at by the title, and students will find the book very readable. Engineering undergraduates encounter devices earlier in their course than do their counterparts in physics, but without the same background. The authors therefore start with a survey of the relevant quantum properties of electrons. They proceed to the electronic properties of materials without getting bogged down in detail, and in my opinion do this rather well.

Richard H. Bube's Electrons in Solids is intended for students of engineering and also materials science. It is subtitled $A n$ Introductory Survey, and in about half the number of pages takes the subject from the same starting point as Solymar and Walsh to a deeper level, with parts that are more appropriate to first-year graduates.

The first five chapters provide a survey of the physics of waves, electromagnetism and atomic structure, with quite a lot of mathematical detail. This may be useful for reference or revision, but I doubt whether many people would start their study of these topics from here. The author then deals with the free-electron theory of metals, band theory, and the optical, electrical and magnetic properties of crystals - I recommend the chapter on optical properties as providing a particularly helpful survey of what is sometimes a confusing topic. The reader is also introduced to a number of different semiconductor devices, including modern ones involving multilayer structures or 'band gap engineering'. This second edition differs from the first only in updating, some changes in presentation and the inclusion of a good number of problems and worked examples.

An Introduction to Solid State Diffusion, by Richard J. Borg and G.J. Dienes, is really in a different category to the other books reviewed here because it concentrates entirely on one topic, the processes by which atoms migrate through crystalline materials. This is an important topic at the interface of physics and chem- istry, being relevant to solid-state reactions, corrosion, and the fabrication and degradation of materials and devices. The book could serve as an introduction to the potential specialist or as a simple reference work for the rest of us. A background knowledge of first-year physical chemistry is assumed, but I feel that those who wil get most from the book will almost certainly have wider experience.

The authors show up the wealth of material in solid-state science by the variety of different cases that have to be considered. But it seems a pity that although their treatment of semi-

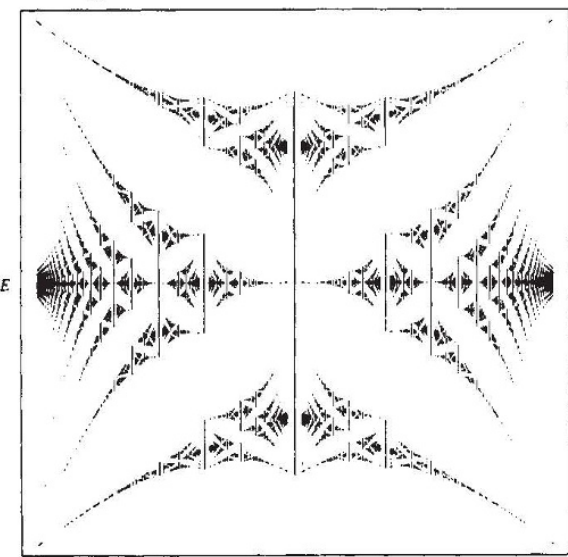

Pattern in physics - an energy diagram for an electron travelling in a weak periodic potential. The diagram appears in A.B. Pippard's Magnetoresistance in Metals, published earlier this year by Cambridge University Press.

conductors includes the concentrations of electrons and holes, the diffusion of these charge carriers (which is so beautifully demonstrated in the Haynes-Shockley experiment) is considered to be outside the scope of the book. It is also unfortunate that, in the attempt to maintain generality while avoiding the use of conventional vector analysis, the theory of diffusion has been based on some strange and inadequately explained equations.

It is intriguing, and surely says something about the state of the subject, that here we have an "Introduction" to one aspect of the solid state that is more than half as long as Christman's "Fundamentals", and yet that aspect is not even mentioned by Christman. Which brings us back to the question of how to design the syllabus for an undergraduate course. Students need the breadth that comes from contact with specialists in more than one area, but they are already being swamped with detail. Each of these books provides a credible structure for courses with a particular emphasis, but the search continues for a text that combines balanced breadth with reliability and simplicity.

R.W. Whitworth is a Senior Lecturer in the School of Physics and Space Research. University of Birmingham, Birmingham B15 $2 \pi$, UK.

\section{Noted approach}

\section{D.J. Raine}

General Relatlvity: A Gulde to Its Consequences for Gravity and Cosmology. By J.L. Martin. Ellis Horwood: 1988. Pp. 176. $£ 32.50$. Distributed in the United States by John Wiley, \$63.95.

THERE is, of course, no ideal book for the course. The standard works are too encyclopaedic, the popular expositions lack mathematical detail, the undergraduate texts are too dull or too idiosyncratic. So, over the years, one develops a set of lecture notes which become handouts to appreciative students, who find them just what they need to answer the examination questions. A publisher is found and another textbook enters the crowded market place.

There are, basically, only two ways to expound general relativity. One starts with the machinery of riemannian geometry, building up to Einstein's equations and their solutions. The other begins with the non-relativistic affine geometry of newtonian gravity, adding the metric when relativity is introduced. Either can come with arbitrary amounts of mathematical leavening.

Dr Martin chooses the first approach. In a book addressed principally to physicists, he keeps the mathematics strictly to a minimum. And he gets to the physics as rapidly as possible by dealing with the Schwarzschild metric and the classical tests of the theory before the curvature tensor and field equations. Then there is additional material on black holes, gravitational collapse, gravitational radiation and some cosmology. Nevertheless, as usual in this approach, Einstein's theory does tend to emerge as a dog wagged by a tail, a theory that just happens to satisfy observations.

But there is a problem - lecture notes are not textbooks. It is not so amusing to read that there is no real evidence for the hot big bang, or to find the equation of state of inflationary models dismissed as being improbable. Should not the student who is interested in physics, and who has worked through a course in general relativity, know the importance of the binary pulsar and be able to recognize the contemporary version of the cosmological constant problem?

Let me emphasize that this is not a bad book; on second reading I began to warm to the attention to detail, the care for students' intellectual digestive capacities, the no-nonsense, clear style. But I wonder how many will think it is the ideal book for their course.

D.J. Raine is a Lecturer in the Department of Astronomy, University of Leicester, Leicester LE1 TRH, UK. 